Research Paper

\title{
High prevalence of SARS-CoV-2 antibodies in care homes affected by COVID-19: Prospective cohort study, England
}

\author{
Shamez N Ladhani ${ }^{\mathrm{a}, \mathrm{b}, *}$, Anna Jeffery-Smith ${ }^{\mathrm{c}}$, Monika Patel ${ }^{\mathrm{c}}$, Roshni Janarthanan ${ }^{\mathrm{d}}$, \\ Jonathan Fok ${ }^{\mathrm{d}}$, Emma Crawley-Boevey ${ }^{\mathrm{d}}$, Amoolya Vusirikala ${ }^{\mathrm{d}}$, \\ Elena Fernandez Ruiz De Olano ${ }^{\mathrm{d}}$, Marina Sanchez Perez ${ }^{\mathrm{d}}$, Suzanne Tang ${ }^{\mathrm{d}}$, \\ Kate Dun-Campbell ${ }^{\mathrm{d}}$, Edward Wynne- Evans ${ }^{\mathrm{d}}$, Anita Bell ${ }^{\mathrm{d}}$, Bharat Patel ${ }^{\mathrm{d}}$, \\ Zahin Amin-Chowdhury ${ }^{a}$, Felicity Aiano ${ }^{a}$, Karthik Paranthaman ${ }^{\mathrm{e}}$, Thomas Ma ${ }^{\mathrm{e}}$, \\ Maria Saavedra-Campos ${ }^{\mathrm{e}}$, Joanna Ellis ${ }^{\mathrm{c}}$, Meera Chand ${ }^{\mathrm{f}}$, Kevin Brown ${ }^{\mathrm{c}}$, Mary E. Ramsay ${ }^{\mathrm{a}}$, \\ Susan Hopkins ${ }^{\mathrm{f}}$, Nandini Shetty ${ }^{\mathrm{c}}$, J. Yimmy Chow ${ }^{\mathrm{d}}$, Robin Gopal ${ }^{\mathrm{c}}$, Maria Zambon ${ }^{\mathrm{c}}$ \\ a Immunisation and Countermeasures Division, Public Health England, London, UK \\ ${ }^{\mathrm{b}}$ Paediatric Infectious Diseases Research Group, St. George's University of London, UK \\ ' Virus Reference Department, Public Health England, London, UK \\ ${ }^{\mathrm{d}}$ London Health Protection Team, National Infection Service, Public Health England, London, UK \\ e Field Service, National Infection Service, Public Health England, UK \\ ${ }^{\mathrm{f}}$ Antimicrobial Resistance and Hospital Acquired Infection Department, Public Health England, London, UK
}

\section{A R T I C L E I N F O}

\section{Article History:}

Received 12 August 2020

Revised 27 September 2020

Accepted 2 October 2020

Available online 6 November 2020

\begin{abstract}
A B S T R A C T
Background: We investigated six London care homes experiencing a COVID-19 outbreak and found high rates of SARS-CoV-2 infection among residents and staff. Here we report follow-up investigations including antibody testing in the same care homes five weeks later.

Methods: Residents and staff in the initial investigation had a repeat nasal swab for SARS-CoV-2 RT-PCR and a blood test for SARS CoV-2 antibodies using ELISA based on SARS-CoV-2 native viral antigens derived from infected cells and virus neutralisation.

Findings: Of the 518 residents and staff in the initial investigation, 186/241 (77.2\%) surviving residents and 208/ $254(81.9 \%)$ staff underwent serological testing. Almost all SARS-CoV-2 RT-PCR positive residents and staff were seropositive five weeks later, whether symptomatic (residents 35/35, 100\%; staff, 22/22, 100\%) or asymptomatic (residents 32/33, 97.0\%; staff 21/22, 95.5\%). Symptomatic but SARS-CoV-2 RT-PCR negative residents and staff also had high seropositivity rates (residents $23 / 27,85.2 \%$; staff $18 / 21,85.7 \%$ ), as did asymptomatic RT-PCR negative individuals (residents $61 / 91,67.0 \%$; staff 95/143, 66.4\%). Neutralising antibody was detected in $118 /$ $132(89.4 \%)$ seropositive individuals and was not associated with age or symptoms. Ten residents (10/79 retested, $12.7 \%$ ) remained RT-PCR positive but with higher RT-PCR cycle threshold values; $7 / 10$ had serological testing and all were seropositive. New infections were detected in three residents and one staff. Interpretation: RT-PCR provides a point prevalence of SARS-CoV-2 infection but significantly underestimates total exposure in outbreak settings. In care homes experiencing large COVID-19 outbreaks, most residents and staff had neutralising SARS-CoV-2 antibodies, which was not associated with age or symptoms. Funding: PHE
\end{abstract}

Crown Copyright $\odot 2020$ Published by Elsevier Ltd. This is an open access article under the CC BY-NC-ND license (http://creativecommons.org/licenses/by-nc-nd/4.0/)

\section{Introduction}

Nursing and residential homes have been disproportionally affected by COVID-19 with high rates of hospitalisations and deaths

\footnotetext{
* Corresponding author at: Immunisation and Countermeasures Division, Public Health England, 61 Colindale Avenue, London NW9 5EQ, UK.

E-mail addresses: shamez.ladhani@phe.gov.uk, drshamez@aol.com (S.N. Ladhani).
}

among residents [1]. In England, the first cases of imported COVID-19 were confirmed in late January 2020 with autochthonous transmission established by early March 2020. Cases peaked in mid-April before declining as a consequence of intense control measures [2]. London, England, was one of the most affected cities and large outbreaks associated with high case fatality rates (CFR) among residents were reported in London care homes [2]. 


\section{Research in Context}

\section{Evidence before this study}

We searched PubMed with the terms "COVID-19 outbreak" or "SARS-CoV-2 outbreak" and "care home", "nursing home", "nursing facility" or "residential home" to identify publications relating to COVID-19 outbreaks since January 2020, focusing particularly on enhanced outbreak investigations and antibody testing. Outbreak investigations of single care homes have identified high rates of asymptomatic and pre-symptomatic SARSCoV-2 infection among residents and staff. There are very limited data on antibody responses to SARS-CoV-2 or the quality of the antibodies in older people.

\section{Added value of this study}

In six London care homes experiencing a large outbreak of COVID-19, 95-100\% of staff and surviving residents who had initially tested positive for SARS-CoV-2 RT-PCR RNA on nasal swab had detectable SARS-CoV-2 antibodies five weeks later. Overall, more than two-thirds of residents and staff members had detectable antibodies against SARS-CoV-2 irrespective of their nasal swab results or symptom status. Neutralising antibodies were present in $89 \%$ of seropositive individuals and were not associated with age, sex, initial nasal swab positivity, presence of symptoms or resident/staff status.

\section{Implications of all the available evidence}

RT-PCR testing for SARS-CoV-2 significantly underestimates the true extent of an outbreak in institutional settings. SARS-CoV-2 seropositivity rates in the care homes affected by COVID-19 were far higher than any healthcare setting, including hospitals, possibly because of the intensity and duration of exposure to the virus within the care home setting. Surveillance is on-going to determine whether SARS-CoV-2 antibodies protect against re-infection and, if so, the duration of protection.

Between 10-13 April 2020, we investigated six London care homes reporting a suspected or confirmed COVID-19 outbreak to Public Health England (PHE) [3]. We found that $40 \%$ of residents (105/264) and $21 \%$ of staff (53/254) had confirmed SARS-CoV-2, with half of both groups remaining asymptomatic throughout the surveillance period [3]. Mass serological testing can help uncover the true extent of an outbreak within the care home setting, [4] and potentially inform staff allocation and cohorting practices. Neutralising antibodies, in addition, may provide evidence for protection against reinfection, especially among the older residents who may not reliably mount an adequate protective response despite antibody production because of immunosenescence [5,6]. As part of follow-up investigations, the residents and staff in the six care homes experiencing a COVID-19 outbreak were followed-up with a repeat nasal swab and a blood sample five weeks after the initial investigations. The study aimed to estimate SARS-CoV2 seropositivity and neutralising antibodies in care home residents and staff of care homes experiencing a COVID-19 outbreak and assess any association with age, symptoms and SARS-CoV-2 reverse transcription polymerase chain reaction (RT-PCR) positivity.

\section{Methods}

We identified six care homes reporting a suspected outbreak $(\geq 2$ suspected cases) of COVID-19 to PHE during 10-13 April 2020 [3]. These were nursing or mixed nursing/residential homes of different sizes, providing care for $43-100$ residents with $14-130$ staff. The care homes were in different stages of a COVID-19 outbreak. During the initial investigation, nasal swabs were taken for SARS-CoV-2 RTPCR for all residents and staff working in the care home at the time. Infection control measures were reinforced and all SARS-CoV-2 RTPCR positive individuals were isolated. All tested participants were followed up for any symptoms during the two weeks before, at the time of testing and for two weeks after the test [3].

Follow-up investigation involved a repeat nasal swab and a blood sample from all participants five weeks after the initial RT-PCR testing. The follow-up investigation protocol was reviewed and approved by PHE Research Ethics and Governance Group and participating care homes. Care home managers obtained verbal consent from staff members and from residents who could give their own consent. Otherwise, their next of kin was contacted with information about the additional testing and asked to provide verbal consent over the phone. Testing began on the week of May 18, 2020. Care home staff took nasal swabs for residents and submitted their own samples by self-swabbing with appropriate instructions. Care home nurses took blood samples from residents and their colleagues, with external phlebotomists assisting two care homes with sampling.

\section{SARS-CoV-2 antibody testing}

SARS-CoV-2 infected virus lysate assay: Native virus antigen ELISA was modified from a previously described MERS-CoV assay [7,8]. Microplate bound detergent (Triton X100) extracted lysates of SARSCoV-2 (isolate England/02/2020) infected Vero E6 cells and uninfected cells were reacted with a serial dilution of convalescent serum obtained from participants in an indirect ELISA format. Virus lysates contained a mixture of viral proteins expressed in Vero E6 cells, including viral nucleocapsid and spike proteins. The reactivity of given sera against virus infected cells and uninfected cells is compared creating a single index value. Sensitivity was determined using convalescent serum samples from SARS-CoV-2 RT-PCR positive individuals at least 21 days after the positive respiratory tract swab [9].

\section{Microneutralisation assay and neutralising antibody titre}

SARS-CoV-2 (isolate England/02/2020) specific neutralising antibody levels were measured using a modification of the WHO influenza microneutralisation methodology [10]. Briefly, 200 TCID 50 of virus was incubated with serial dilutions of serum from participants, after which a suspension of Vero E6 cells were added. After 22 h, cells were fixed and in-cell SARS-CoV-2 nucleoprotein (NP) expression determined by ELISA using Rabbit polyclonal to SARS-CoV Nucleoprotein (SinoBiological; Cat no 40143-T62).

The virus neutralising antibody titre was determined as the serum concentration that inhibited 50\% SARS-CoV-2 NP expression. All work was undertaken in a BSL-3 laboratory.

\section{SARS-COV-2 PCR}

Nucleic acid was extracted from samples and analysed by a realtime RT-PCR assay targeting a conserved region of the open reading frame $1 \mathrm{ab}(\mathrm{ORF} 1 \mathrm{ab})$ gene of SARS-CoV-2, together with detection of an assay internal control to monitor the extraction and RT-PCR processes. This assay used the primers and probe sequences made public by CDC China (http://ivdc.chinacdc.cn/kyjz/202001/t20200121_ 211337.html) and required $5 \mu$ L RNA in a total RT-PCR reaction volume of $25 \mu \mathrm{L}$. Reverse transcription and PCR amplification was performed on an Applied Biosystems 7500 FAST system.

\section{Statistical analysis}

Descriptive analyses were performed. Data that did not follow a normal distribution were described as medians with interquartile ranges and compared using the Mann-Whitney U test. Antibody 

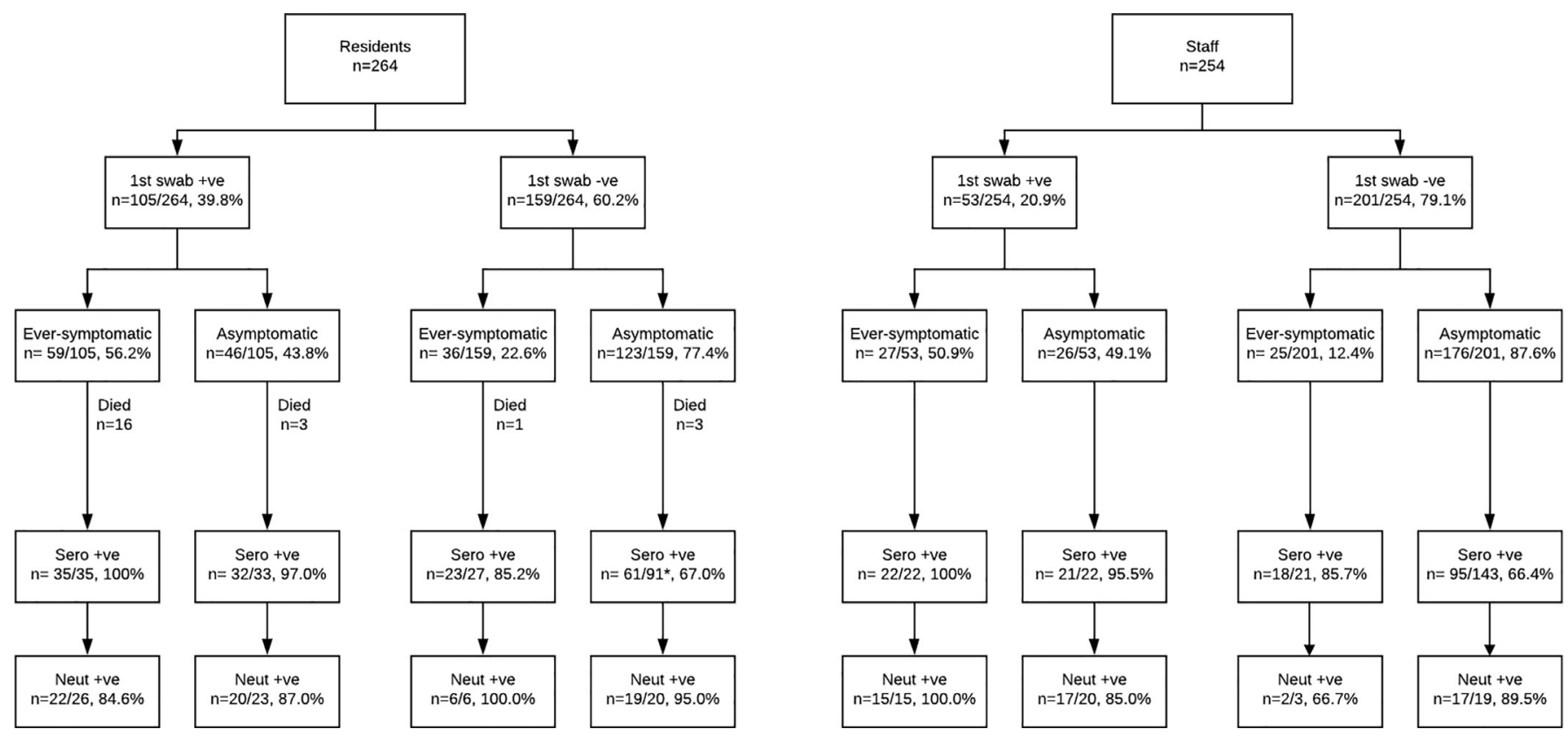

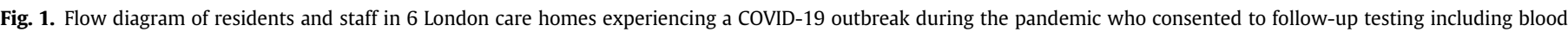
sampling for SARS-CoV-2 antibodies four to six weeks later. 'Ever-symptomatic' indicates that symptoms were experienced at some point during the follow-up period.

*Three individuals in this group became SARS-CoV-2 PCR positive at follow-up RT-PCR testing conducted simultaneously with SARS-CoV-2 antibody testing.

concentrations were presented as ELISA index values with medians and $95 \%$ confidence intervals $(95 \% \mathrm{CI})$. Antibody concentrations above the index value of 0.5 were considered positive. Median antibody concentrations were compared using Kruskal-Wallis with Dunn's multiple comparisons test adjustment. Categorical variables were described as proportions and compared using Chi -square or Fisher's Exact test as appropriate. Data were analysed using Stata version 15.0 (Statcorp, Tx) and GraphPad Prism.

Ethics approval: The research protocol was approved by the PHE Research Ethics and Governance Group (REGG Ref: NR0204, 07 May 2020).

Role of the funding source: This study was funded by Public Health England as part of the COVID-19 response. The authors had sole responsibility for the study design, data collection, data analysis, data interpretation, and writing of the report. The authors are all employed by Public Health England, the study funder, which is a public body - an executive agency of the Department of Health. SNL and MZ had full access to all the data in the study and final responsibility for the decision to submit for publication.

\section{Results}

\section{Seropositivity}

Of the original 518 residents and staff involved in the initial care home outbreak investigation during 10-13 April 2020, 394 (76.1\%) consented for follow-up investigations at median of 36 days (range, 30-45 days) and were tested using the virus lysate antibody assay (Fig. 1). SARS-CoV-2 seropositivity for the cohort was $77.9 \%(95 \% \mathrm{CI}$, 73.6-81.7\%; Table 1, Fig. 2a). Seropositivity was associated with being symptomatic and SARS-CoV-2 RT-PCR nasal swab positive at the initial test (Fisher's exact test; both $p<0.0001$ ), but not with gender or being a resident or staff member (Table 1, Fig. 2).

\section{Residents}

Twenty-one of the 264 residents tested in the initial investigations died within two weeks and two others died prior to follow-up testing.
Thus, 186 of the remaining 241 residents consented to SARS-CoV-2 antibody testing and $81.2 \%(151 / 186)$ were seropositive. Of the 186 residents for who convalescent serological analysis was available 35 had been symptomatic and RT-PCR positive during the initial testing period; all were SARS-CoV-2 antibody positive (Fig. 1, Fig. 2c). For the residents who were RT-PCR positive but remained asymptomatic throughout the outbreak, 97.0\% (32/33) were SARS-CoV-2 antibody positive (Fig. 1, Fig. 2c). Of the 118 residents with convalescent serology who had tested RT-PCR negative initially, seropositivity was $85.2 \%$ (23/27) in those who had been symptomatic during the outbreak and $67.0 \%$ (61/91) in residents who remained asymptomatic (Fig. 1, Fig. 2c).

\section{Staff}

Among the 254 staff members involved in the initial investigation, 208 consented to additional investigations and 75.0\% (156/208) were seropositive (Fig. 2a). All of those who were SARS-CoV-2 RT-PCR positive at initial testing and symptomatic during the outbreak were positive for SARS-CoV-2 antibodies (22/22), as were 95.5\% (21/22) of RTPCR positive asymptomatic staff (Fig. 1, Fig. 2c). Of the 164 staff members with convalescent serology who were SARS-CoV-2 RT-PCR negative at the initial test time point 21 experienced COVID-19 compatible symptoms during the follow-up period and 18 (85.7\%) were SARS-CoV-2 antibody positive. The remaining 143 SARS-CoV-2 PCR negative staff undergoing serological analysis remained asymptomatic throughout the surveillance period; 95 (66.4\%) were SARSCoV-2 antibody positive (Fig. 1, Fig. 2c).

\section{SARS-CoV-2 antibody seropositivity and index values}

There was no association between SARS-CoV-2 seropositivity and age (Chi-square test, $P=0.43$, Table 1 ) (Fig. 3, left panel). Among SARS-CoV-2 antibody positive individuals, there was no significant difference in median index value between the those who had been PCR positive or negative at initial testing (Mann-Whitney U Test $P=0.05$; Supplementary figure $1 \mathrm{a}$ ) or by age (Kruskal Wallis with Dunn's multiple comparisons test $P=0.07$; Fig. 3, right panel). There was no association between median SARS-CoV-2 antibody index 
Table 1

Demographics of care homes cohort and seropositivity by group: percentage and $95 \%$ confidence intervals shown. Statistical analysis using Fisher's exact test ${ }^{(\mathrm{f})}$ and Chisquare test of proportions ${ }^{(\mathrm{c})}$, $\mathrm{p}$ values as shown.

\begin{tabular}{|c|c|c|c|c|c|}
\hline & $\mathrm{n}$ & $\%$ total & Seropositive (n) & $(\%)$ & $95 \% \mathrm{CI}$ \\
\hline $\begin{array}{l}\text { Overall } \\
\text { Sex }\end{array}$ & 394 & 100 & 307 & 77.9 & $73.6-81.7$ \\
\hline Male & 95 & 24.1 & 73 & 76.8 & $67.4-84.2$ \\
\hline $\begin{array}{l}\text { Female } \\
p \text { value }{ }^{(f)} \\
\text { Age group }\end{array}$ & 299 & 75.9 & 234 & $\begin{array}{l}78.3 \\
0.78\end{array}$ & $73.2-82.6$ \\
\hline $19-39$ & 66 & 16.8 & 49 & 74.2 & $62.6-83.3$ \\
\hline $40-59$ & 113 & 28.7 & 88 & 77.9 & $69.4-84.5$ \\
\hline $60-79$ & 71 & 18.0 & 52 & 73.2 & $61.9-82.1$ \\
\hline $\begin{array}{l}>80 \\
p_{\text {v value }}^{(\mathrm{c})}\end{array}$ & 144 & 36.5 & 118 & $\begin{array}{l}81.9 \\
0.43\end{array}$ & $74.9-87.4$ \\
\hline \multicolumn{6}{|c|}{ Residents and Staff } \\
\hline Residents & 186 & 47.2 & 151 & 81.2 & $75.0-86.1$ \\
\hline $\begin{array}{l}\text { Staff } \\
\text { p value }^{(f)}\end{array}$ & 208 & 52.8 & 156 & $\begin{array}{l}75.0 \\
0.15\end{array}$ & $68.7-80.4$ \\
\hline \multicolumn{6}{|c|}{ Symptoms at any time during first test period } \\
\hline Symptomatic & 105 & 26.6 & 98 & 93.3 & $86.9-96.7$ \\
\hline $\begin{array}{l}\text { Asymptomatic } \\
\text { p value }^{(\mathrm{f})}\end{array}$ & 289 & 73.4 & 209 & $\begin{array}{l}72.3 \\
<0.0001\end{array}$ & $66.9-77.2$ \\
\hline \multicolumn{6}{|c|}{ First round PCR results } \\
\hline 1st PCR + & 112 & 28.4 & 110 & 98.2 & $93.7-99.7$ \\
\hline $\begin{array}{l}\text { 1st PCR - } \\
\text { p value }^{(f)}\end{array}$ & 282 & 71.6 & 197 & $\begin{array}{l}69.9 \\
<0.0001\end{array}$ & $64.3-74.9$ \\
\hline
\end{tabular}

value and symptom status (Mann-Whitney U Test $P=0.96$; Supplementary figure $1 \mathrm{~b}$ ), or gender (Mann-Whitney U Test $P=0.57$; Supplementary figure 1c). Six individuals had equivocal SARS-CoV-2 antibody index values; all were asymptomatic throughout the surveillance period and were SARS-CoV-2 RT-PCR negative on nasal swabs at both timepoints.

\section{Neutralising antibodies}

Neutralising antibodies were detected in $89.4 \%$ (118/132) of seropositive individuals. There was no association between the detection of SARS-CoV-2 neutralising antibodies and age (Chi-square test $P=0.27$; Fig. $4 \mathrm{a}$ ) or PCR status (Fisher's exact test $P=0.77$ ); data not shown. There was no significant difference in neutralising antibody titre by sex (Mann-Whitney $U$ test $P=0.69$; Fig. 4 b), or symptom status (Mann-Whitney U test $P=0.10$; Fig. $4 \mathrm{c}$ ). There was a trend toward increasing neutralising antibody titre with age (Kruskal-Wallis test with Dunn's Multiple comparisons, $P=0.40$; Fig. 4d).

\section{Nasal swab RT-PCR}

All consenting residents and staff had a repeat nasal swab at the time of convalescent blood sampling. Thirteen residents were SARSCoV-2 RT-PCR positive on this repeat sample, including 10 who had been SARS-CoV-2 RT-PCR positive at an interval of 36-45 days previously, although SARS-CoV-2 RT-PCR Ct values were significantly lower at follow-up (Supplementary Figure 2). Of these ten, 7 consented to serum sampling and all were seropositive with neutralising antibodies detected in all of those tested (Supplementary Table 1). Three residents became SARS-CoV-2 RT-PCR positive at follow-up and all were seropositive (Fig. 1); all remained asymptomatic. None of the staff who were SARS-CoV-2 RT-PCR positive at the initial visit were positive on repeat testing. A previously SARS-CoV-2 RT-PCR negative staff member who remained asymptomatic throughout the surveillance period became RT-PCR positive on repeat testing, with a $\mathrm{Ct}$ value of 35.6; this staff member was seropositive for SARS-CoV-2 antibodies.

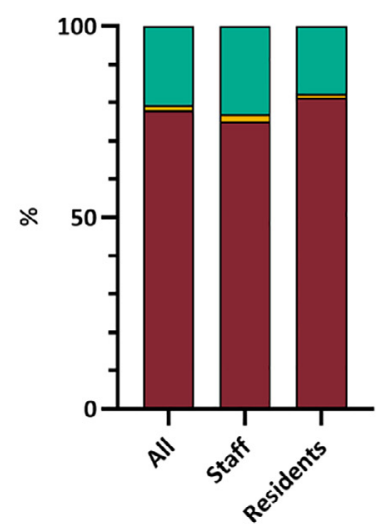

b

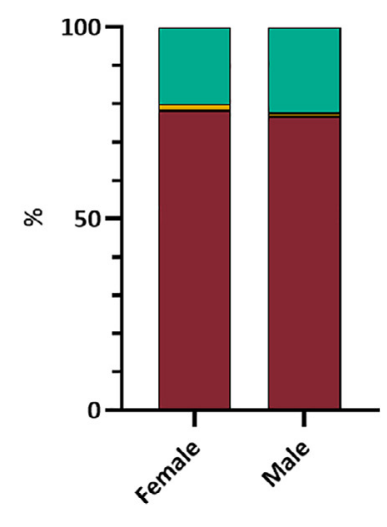

C

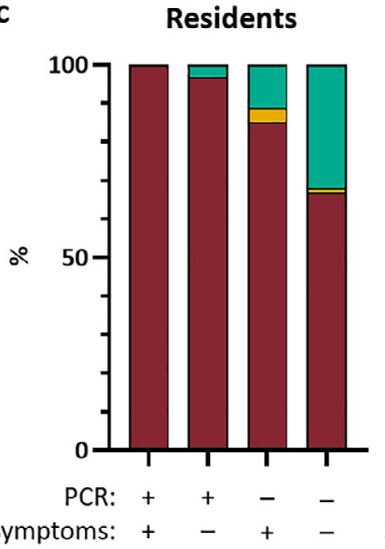

Staff

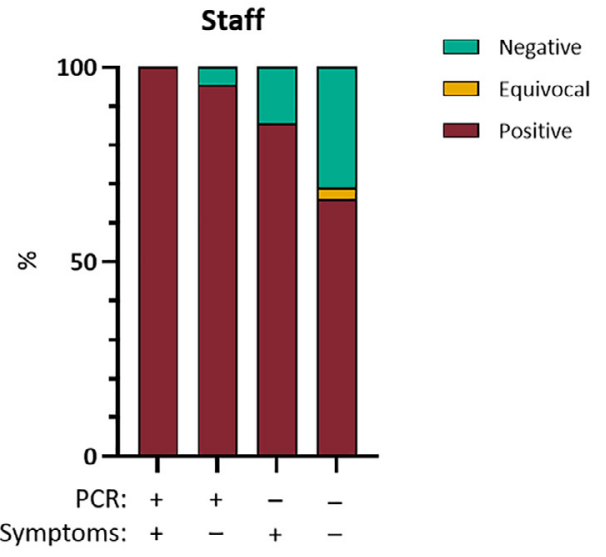

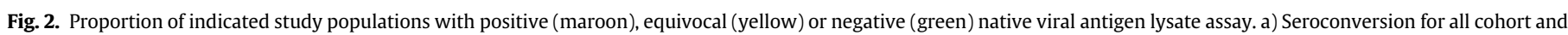

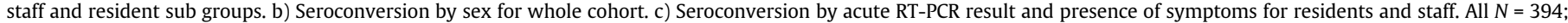
Staff $N=208$; Residents $N=186$. 

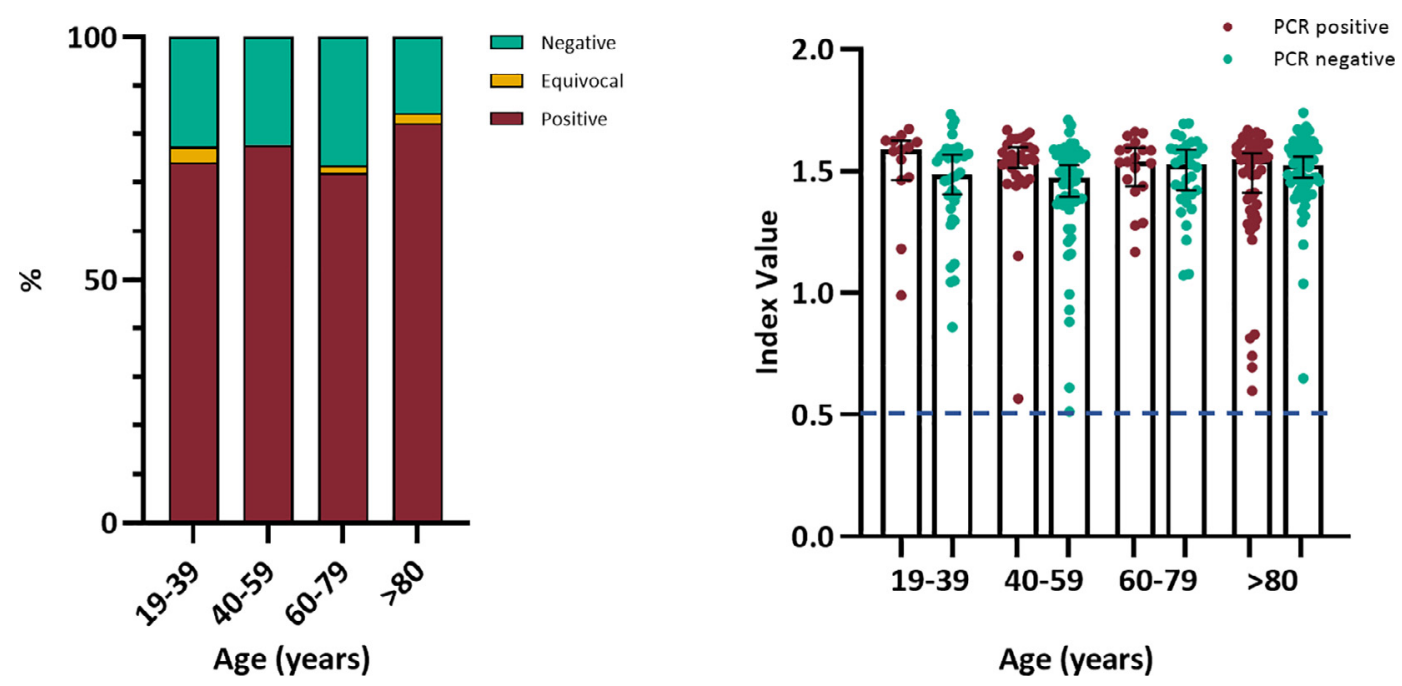

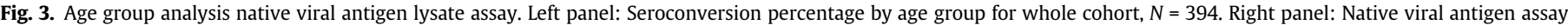

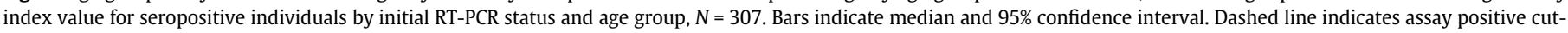
off. Statistical analysis using Chi-square test of proportion $(p=0.43)$ and Kruskal-Wallis with Dunn's multiple comparisons test $(p=0.07)$.
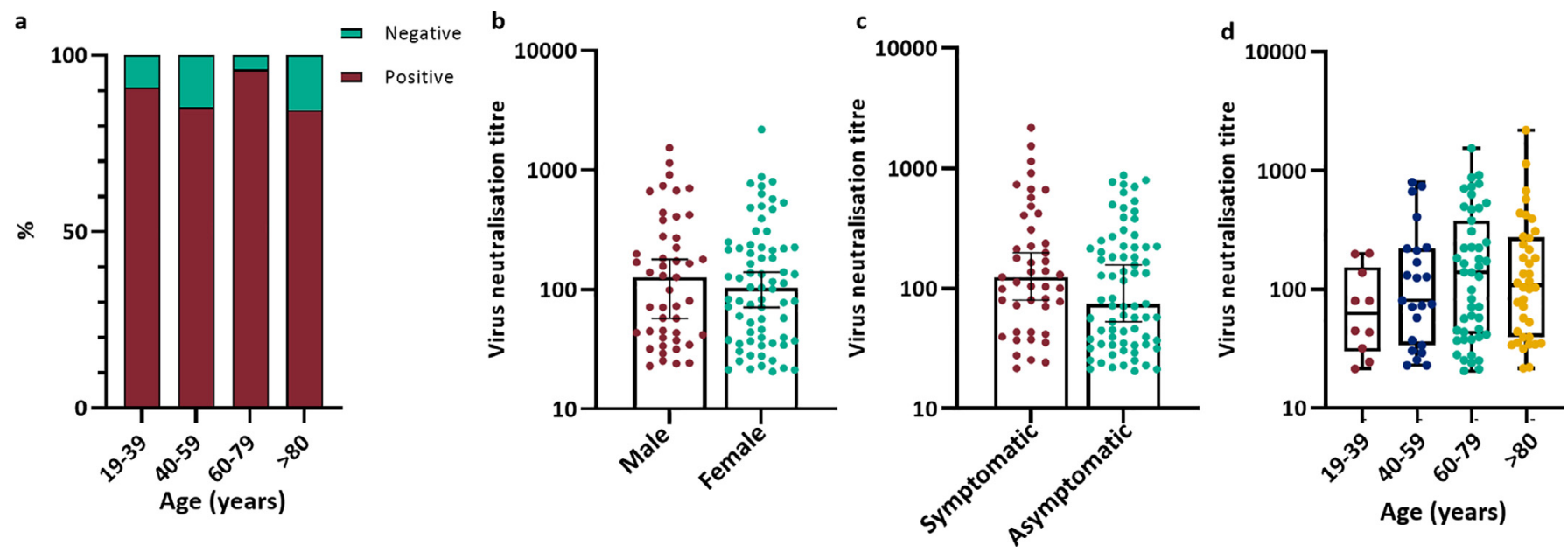

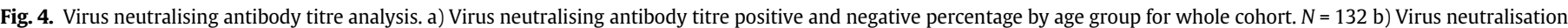

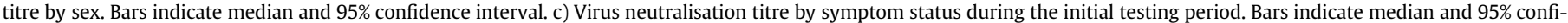

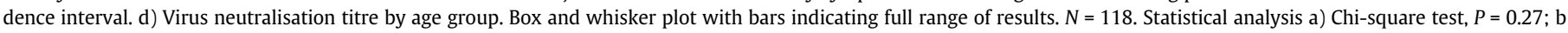
Mann-Whitney U Test, $P=0.69$; c) Mann-Whitney U Test, $P=0.10$ d) Kruskal Wallis with Dunn's multiple comparisons test adjustment, $P=0.40$.

\section{Discussion}

In six London care homes experiencing a COVID-19 outbreak at the peak of the pandemic, $81.2 \%$ of surviving residents and $75.0 \%$ of staff were SARS-CoV-2 antibody positive five weeks after the initial outbreak investigation. These rates are far higher than those reported from any other cohort including frontline healthcare workers managing patients with confirmed COVID-19 in hospitals [11-13]. Almost all residents and staff who were SARS-CoV-2 RT-PCR positive on nasal swab at initial testing developed SARS-CoV-2 antibodies, irrespective of whether they were symptomatic at any point during the outbreak. High seropositivity rates were also observed for symptomatic residents and staff even if they had a previously negative SARS-CoV-2 RT-PCR test. The serological investigation emphasises the extent to which SARS-CoV-2 can penetrate vulnerable communities in closed settings, and the underestimation of exposure through point prevalence estimates using RT-PCR from nasal swabs. The lowest seroprevalence was observed in residents and staff who remained asymptomatic throughout the outbreak and who were SARS-CoV-2 RT-PCR negative on both testing visits but, even in this group, more than two-thirds were positive for SARS-CoV-2 antibodies. In this cohort, SARS-CoV-2 antibody levels were not associated with age, sex, presence of symptoms, PCR-positivity or resident/staff status.

There are now sensitive and specific serological assays, such as the one used in this investigation based on using native viral antigens derived from infected cells [9]. Overall, a recent systematic review estimated that serological tests had $30 \%$ sensitivity for SARS-CoV-2 antibodies during the first week after symptom onset, rising to $72 \%$ in the second week, 91\% in the third week and $96 \%$ up to 5 weeks later [14]. The finding that almost all residents and staff with confirmed COVID-19 through nasal swab SARS-CoV-2 RT-PCR - irrespective of whether they ever experienced symptoms during the outbreak - is reassuring and validates the use of our serological assay as a measure of past exposure. The very high seropositivity rates among symptomatic but RT-PCR negative residents and staff suggests that their original illness was also most likely due to COVID-19 and highlights the sensitivity limitations of single point nasal swabbing for diagnosis and the narrow window of SARS-CoV-2 detection in infected individuals. RT-PCR significantly underestimated exposure to SARS-CoV-2 in these outbreak settings [15]. The very high seropositivity of $75.0 \%$ among care home staff compared to $17-44 \%$ of patient-facing healthcare workers is staggering [11,12]. A possible 
explanation may be more prolonged and intense exposure to the virus because of level of care required by the residents $[15,16]$. In our initial investigations we also found evidence of transmission between staff members in care homes, highlighting the importance of maintaining infection control practices for all contact, including those between staff, whilst on care home premises [8]. Despite reinforcement of infection control measures at the outset, one further staff member and three residents became infected with SARS-CoV-2 at follow-up. Residents and staff who were SARS-CoV-2 RT-PCR positive at follow-up all had high $\mathrm{Ct}$ values, consistent with non-viable virus at the time of testing, [3] and were also SARS-CoV-2 antibody positive. Further studies are needed to assess whether the presence of SARSCoV-2 antibodies, including neutralising antibodies, are protective against re-infection and, if so, the duration of protection. Questions also remain as to whether antibody concentrations provide a useful measure of protection in infected individuals [17].

Interestingly, SARS-CoV-2 antibody levels were similar among symptomatic and asymptomatic residents and staff across the care homes [13]. The high fatality rates among residents across the six care homes, particularly affecting those who had been symptomatic and SARS-CoV-2 RT-PCR positive, indicates that the cohort described here is more representative of milder illness and depleted of individuals who suffered the most severe outcomes of infection [3]. We also found that $90 \%$ of seropositive staff and residents had neutralising antibody responses, with no significant differences in neutralising antibody levels between by age, sex, symptom status, or staff/resident status. There was a trend towards increasing neutralising antibody titres with increasing age (Fig. 4c) but this was not statistically significant. These findings of such robust antibody responses in surviving care home residents, especially when compared to younger, healthier staff members with similar exposure to SARS-CoV-2, are novel and may play an important part in future recommendations for infection control practices and vaccination against SARS-CoV-2.

Several key questions relating to this novel pandemic remain to be answered and are particularly relevant to this highly vulnerable population and setting. In particular, it is not known whether SARSCoV-2 antibodies are protective against re-infection [17]. We identified a small number of residents who were still RT-PCR positive up to five weeks later; all of those tested had detectable antibodies, including neutralising antibodies at the time of the persistent virus detection [18]. The RT-PCR Ct values were consistent with non-live virus in all residents and staff members who were RT-PCR positive on nasal swab at follow-up. The prolonged nasal swab RT-PCR positivity in a proportion of residents and staff is consistent with a recent large healthcare worker study where up to a quarter were still RT-PCR positive up to six weeks later, highlighting yet another limitation of our understanding of the kinetics of viral replication and immune responses COVID-19 $[19,20]$. This well-characterised cohort will be followed with routine care home screening, [21] providing key insights into the potential for reinfection in seropositive individuals. Subsequent serology follow-up is planned for this cohort to explore the longevity of serological responses.

The strengths of our investigations include the extensive and robust epidemiological, virological and serological testing of residents and staff across six London care homes experiencing large outbreaks of COVID-19, the broad age ranges involved, the daily followup after initial testing and the high uptake for retesting five weeks later. The data collected have provided a wealth of information on SARS-CoV-2 infection, transmission and antibody responses in a high-risk care setting involving a very vulnerable cohort. A limitation is that the care homes were already in the middle of the outbreak. Consequently, some residents had already developed COVID-19 and some had died of their infection. Another limitation was that we did not obtain blood samples for antibody testing at the first visit, which could have provided additional useful information on antibody kinetics in a large cohort of elderly residents and younger staff members.
The lower nasal swab positivity during the initial investigations compared to the antibody results five weeks later reflects the limited sensitivity of the test, the quality of sampling, the stage of infection at the time of testing and the gene targets used by different RT-PCR assays [22]. Some of these limitations could potentially have been mitigated by repeated swabbing at different time points. Finally, a quarter of the residents and staff in the initial investigations were not included in the follow-up, either because it was not possible to get informed consent from the residents or their next of kin, or because the staff were not working at the care home when follow-up investigations were performed.

In conclusion, almost all residents and staff with confirmed SARSCoV-2 infection had detectable antibodies five weeks later, irrespective of whether they were ever symptomatic or remained asymptomatic throughout the outbreak. Additionally, a high proportion of those who were symptomatic but SARS-CoV-2 RT-PCR negative were also seropositive. SARS-CoV-2 antibody levels were not associated with age, sex, PCR positivity, symptom status or resident/staff status. Our findings demonstrate that older and vulnerable residents are able to mount a robust antibody response to SARS-CoV-2 that is similar to younger healthy staff members. This study highlights the value of serological analysis in addition to RT-PCR screening to understand SARS-CoV-2 exposure in this high-risk population, which is vital for informing winter-planning and vaccine strategies. Further studies are needed to determine whether SARS-CoV-2 antibodies protect against re-infection and, if so, the duration of protection.

\section{Declaration of Competing Interest}

The authors have nothing to declare.

\section{Acknowledgements}

The authors are very grateful to the care home managers, their staff and the residents for their willingness to support the investigation, along with the staff in the immunisation and countermeasures division, PHE Operations, the virus reference department, the London Coronavirus Response Cell and Field Services for their help and support with the investigation.

\section{Data sharing statement}

The data collected were the part of enhanced outbreak investigations duties by Public Health England. There are no additional data to share. SNL and MZ had full access to all the data in the study and final responsibility for the decision to submit for publication.

\section{Funding}

Public Health England.

\section{Supplementary materials}

Supplementary material associated with this article can be found, in the online version, at doi:10.1016/j.eclinm.2020.100597.

\section{References}

[1] Office for National Statistics (ONS). Deaths involving COVID-19 in the care sector England and Wales: deaths occurring up to 12 June 2020 and registered up to 20 June 2020 (provisional). 03 July 2020. (Accessed 04 September 2020, 2020. Available at: https://www.ons.gov.uk/peoplepopulationandcommunity/birthsdeathsandmarriages/deaths/articles/deathsinvolvingcovid19inthecaresectorenglandandwales/deathsoccurringupto12june2020andregisteredupto20june2020provisional. Accessed: 05 September 2020.

[2] Public Health England. National COVID-19 surveillance reports 2020. Available at: https://www.gov.uk/government/publications/national-covid-19-surveillancereports. Accessed: 05 September 2020. 
[3] Ladhani SNC, Chow JY, Janarthanan R, et al. INVESTIGATION of SARS-CoV-2 outbreaks in six care homes in London. The London Care Home Investigation; 2020 APRIL 2020EClinicalMedicine(in press).

[4] Lerner AM, Eisinger RW, Lowy DR, et al. The COVID-19 serology studies workshop: recommendations and challenges. Immunity 2020 Available at: https:/ www.cell.com/immunity/pdf/S1074-7613(20)30267-3.pdf Accessed: 05 September 2020.

[5] Nikolich-Zugich J, Knox KS, Rios CT, Natt B, Bhattacharya D, Fain MJ. SARS-CoV-2 and COVID-19 in older adults: what we may expect regarding pathogenesis, immune responses, and outcomes. Geroscience 2020;42:505-14.

[6] Bencivenga L, Rengo G, Varricchi G. Elderly at time of COronaVIrus disease 2019 (COVID-19): possible role of immunosenescence and malnutrition. Geroscience 2020 Available at: https://link.springer.com/content/pdf/10.1007\%2Fs11357020-00218-9.pdf Accessed: 05 September 2020.

[7] Agnihothram S, Gopal R, Yount Jr. BL, et al. Evaluation of serologic and antigenic relationships between middle eastern respiratory syndrome coronavirus and other coronaviruses to develop vaccine platforms for the rapid response to emerging coronaviruses. J Infect Dis 2014;209:995-1006.

[8] Harvey R, Mattiuzzo G, Hassall M, et al. Comparison of serologic assays for middle east respiratory syndrome coronavirus. Emerg Infect Dis 2019:25:1878-83.

[9] Harvala H, Mehew J, Robb ML, et al. Convalescent plasma treatment for SARS CoV-2 infection: analysis of the first 436 donors in England, 22 April to 12 May 2020. Euro Surveill 2020;25.

[10] Serological diagnosis of influenza by microneutralization assay 6 December 2010 Available at: https://www.who.int/influenza/gisrs_laboratory/2010_12_06_serological_diagnosis_of_influenza_by_microneutralization_assay.pdf. Accessed: 05 September 2020.

[11] Chen Y, Tong X, Wang J, et al. High SARS-CoV-2 antibody prevalence among healthcare workers exposed to COVID-19 patients. J Infect 2020 Available at: Accessed: 05 September 2020.

[12] Korth J, Wilde B, Dolff S, et al. SARS-CoV-2-specific antibody detection in healthcare workers in Germany with direct contact to COVID-19 patients. J Clin Virol 2020;128:104437.
[13] Seow JG C, Merrick B; , et al. Longitudinal evaluation and decline of antibody responses in SARS-CoV-2 infection. MedRxiv 2020 Available at: Accessed: 05 September 2020.

[14] Deeks JJ, Dinnes J, Takwoingi Y, et al. Antibody tests for identification of current and past infection with SARS-CoV-2. Cochrane Database Syst Rev 2020;6: CD013652 Available at: https://www.journalofinfection.com/article/S0163-4453 (20)30344-3/fulltext Accessed: 05 September 2020.

[15] Gordon AL, Goodman C, Achterberg W, et al. Commentary: COVID in care homeschallenges and dilemmas in healthcare delivery. Age Ageing 2020 Available at: https://academic.oup.com/ageing/article/49/5/701/5836695 Accessed: 05 September 2020

[16] Burki T. England and Wales see 20000 excess deaths in care homes. Lancet 2020;395:1602.

[17] Altmann DM, Douek DC, Boyton RJ. What policy makers need to know about COVID-19 protective immunity. Lancet 2020;395:1527-9.

[18] Graham N, Junghans C, Downes R, et al. SARS-CoV-2 infection, clinical features and outcome of COVID-19 in United Kingdom nursing homes. J Infect 2020 Available at: https://www.journalofinfection.com/article/S0163-4453(20)30348-0/ fulltext Accessed: 05 September 2020.

[19] Lombardi A, Consonni D, Carugno M, et al. Characteristics of 1573 healthcare workers who underwent nasopharyngeal swab testing for SARS-CoV-2 in Milan, Lombardy, Italy. Clin Microbiol Infect 2020 Available at: Accessed: 05 September 2020.

[20] Eyre RL SF, O'Donnell D, et al. Differential occupational risks to healthcare workers from SARS-CoV-2: a prospective observational study. MedRxiv 2020 Available at: https://www.medrxiv.org/content/10.1101/2020.05.07.20094276v1 Accessed: 05 September 2020.

[21] Regular retesting rolled out for care home staff and residents. 3 July 2020. Available at: https://www.gov.uk/government/news/regular-retesting-rolled-out-forcare-home-staff-and-residents. Accessed: 05 September 2020.

[22] Watson J, Whiting PF, Brush JE. Interpreting a covid-19 test result. BMJ 2020;369: m1808. Available at: https://www.bmj.com/content/369/bmj.m1808 Accessed: 05 September 2020 